\title{
Visual and tactual texture perception: Intersensory cooperation
}

\author{
MORTON A. HELLER \\ Winston-Salem State University, Winston-Salem, North Carolina 27110
}

\begin{abstract}
In three experiments, subjects were required to make texture judgments about abrasive surfaces. Touch and vision provided comparable levels of performance when observers attempted to select the smoothest of three surfaces, but bimodal visual and tactual input led to greater accuracy. The superiority of bimodal perception was ascribed to visual guidance of tactual exploration. The elimination of visual texture cues did not impair bimodal performance if vision of hand movements were permitted. It is suggested that touch may preempt vision when both sources of texture information are simultaneously available. The results support the notion that perception is normally multimodal, since restriction of the observer to either sense in isolation produces lower levels of performance.
\end{abstract}

In 1891, Fraser bemoaned both the bias toward visual research in psychology and the lack of investigation of the sense of touch. It has often been assumed that people are primarily visual and that the other senses are of secondary importance, and even that vision is better than touch and a "higher" sense, as Thomas Reid claimed when he said, "Of the five senses, sight is without doubt the noblest. The evidence of reason is called seeing, not feeling, smelling, or tasting"' (Reid, 1764/1967, chap. 6, section 1). Lately, however, a more balanced viewpoint has been presented. Advocates of a modality-appropriateness hypothesis have claimed that vision may excel at some tasks, while touch may be better suited to others. A number of researchers have concluded that, while vision is more appropriate for form perception, touch may provide comparable accuracy when less demanding requirements are made upon the observer (Freides, 1974; Kaufman, 1974; Welch \& Warren, 1980).

A rather provocative point of view postulates that touch is more a "reality sense" than vision. In 1749 , Diderot remarked that the sense of touch was responsible for our belief in the existence of objects and educates vision in object permanence (Diderot, $1749 / 1972)$. Reid (1764/1967) proposed that vision must learn to perceive those attributes-hardness and softness, size, motion, and texture-that are originally known directly to the tactual sense. Fraser

\footnotetext{
Some of the results of Experiment 1 were presented at the annual meeting of the Cognition Group of North Carolina in Raleigh, November 1, 1980. I would like to thank Jeff Ersoff, Howard Passel, Faith Heller, Martha Teghtsoonian, and two reviewers for their helpful comments on earlier versions of this manuscript. I wish to thank Brenda A. Boone for assistance with running the experiment described in Footnote 1. Send reprint requests to Morton A. Heller, Psychology, Winston-Salem State University, Winston-Salem, North Carolina 27110.
}

(1891) held that the real world was not a world of reflection, but a world of touch. Recently, Taylor, Lederman, and Gibson (1974) have revived the assertion that something touched is somehow more real than something seen. They have further argued that perceptual activity promotes this sense of "reality" and that multimodal input encourages stable percepts. Von Fieandt (1958) proffers a similar point of view: "The phenomenal object gains in reality when the impression is multidimensional. The material in question must not only offer itself optically, but also preferably tangibly." Neisser (1976) has questioned the ecological validity of research that is limited to just one modality and criticized the lack of multimodal research.

The following three experiments were designed to explicate intermodality relations in the perception of texture. Experiment 1 used relative smoothness judgments to evaluate the effects of multimodal input on texture perception. This experiment also compared vision and touch in the accuracy of smoothness detection. Experiments 2 and 3 addressed the problem of the relationships between the senses in relatively "normal" circumstances. Which cues might be utilized? What were the roles of visual and auditory cues in texture perception? Experiment 3 explored the question of the nature of the visual information that might assist touch in making texture judgments. Was vision used to control hand movements and guide the hand in exploratory activity? An alternative possibility was that information from a secondary sensory channel would serve to aid perceptual accuracy by the provision of redundant input.

\section{EXPERIMENT 1}

A first experiment was performed to examine the effect of combined visual and tactual input on tex- 
ture perception. The subjects explored surfaces with vision alone, touch alone, or both vision and touch. The task required subjects to determine which of three samples of sandpaper was the smoothest. Bimodal input was expected to yield higher levels of accuracy. It was not known whether touch and vision would prove equally accurate in making relative smoothness judgments. Rudel and Teuber (1964) proposed that touch could be superior for texture perception, while Gibson $(1962$, p. 488) commented that the physical texture of a surface could be perceived by either sense alone.

\begin{abstract}
Method
Subjects. The subjects were 48 adults recruited from an undergraduate college population. Half of the subjects were male and half were female. All subjects had normal vision or vision that was corrected to normal.

Stimuli and Apparatus. The stimuli were $5-\mathrm{cm}^{2}$ samples of aluminum oxide abrasive papers manufactured by Norton (Adolox Nofil), with grit values of $80,100,120,150,180,220,240,280$, 320 , and 400 . The grit value reflects the number of openings per inch in a screen that is used to sort the abrasives (Stevens \& Harris, 1962). The smaller the number, the larger the particles and, therefore, the rougher the surface should feel. The stimuli were arranged in series of three adjacent grit values, on rectangular panels $16.5 \times 7.5 \mathrm{~cm}$, their left-right position assigned randomly. There were eight series: $400,320,280 ; 320,280,240$; and so on, to $120,100,80$. A second stimulus set was identical to the first except for different randomly determined positions. The papers were mounted with pressure-sensitive adhesive and were frequently changed. The subjects wore cotton work gloves, with the index finger of the appropriate glove removed, restricting them to the use of the tip of the index finger of the preferred hand. Intramodal vision subjects wore regular work gloves on both hands. Vision was excluded in the haptic condition by a Masonite baffle with an opening at the base covered by an opaque black cloth. Lighting was provided by overhead fluorescent fixtures.

Design and Procedure. The experiment was a mixed-factorial design, the factors being perceptual mode (vision vs. touch vs. bimodal input) and intertrial interval (ITI) ( 0 vs. $1 \mathrm{~min})$, with repeated measures on the second factor. ITI was manipulated to determine if rest periods between trials would facilitate tactual performance, since Walker (1966) reported adaptation effects with tactual exposures to sandpaper. When exploring the stimuli with the preferred hand, all subjects were instructed to pick up and hold each panel by its edges, using the nonpreferred, gloved hand. No time limits were imposed, and the subjects were allowed to repeatedly examine the three surfaces before indicating the smoothest. The relative smoothness judgment was decided upon to permit texture judgments while preventing subjects from basing their responses upon brightness or color. A naive subject cannot know if a brighter surface is rougher or smoother. When vision was permitted, the subjects were encouraged to look as closely as they wished. Half of the subjects in each of the three perceptual mode conditions started with a 0 -sec ITI, and the other half began with a 1-min ITI; all finished with the other ITI condition. The subjects in the haptic group were also asked to hold the panels with their completely gloved hands but with vision obscured. The vision subjects were instructed to point to their choices but not to touch them. Haptic and bimodal subjects were asked to tap the smoothest of the three papers on each trial. No feedback was given.
\end{abstract}

\section{Results and Discussion}

Bimodal visual and haptic input resulted in superior texture judgments (see Table 1). Rest periods
Table 1

Mean Number Correct and Standard Deviation for Each Modality

\begin{tabular}{lcccc}
\hline \multirow{2}{*}{$\begin{array}{c}\text { Perceptual } \\
\text { Modality }\end{array}$} & \multicolumn{3}{c}{ Mean Number Correct } & \\
\cline { 2 - 4 } & 0 ITI & 1-Min ITI & Total & SD \\
\hline Touch & 5.94 & 5.87 & 11.81 & 2.01 \\
Vision & 5.44 & 5.63 & 11.07 & 1.95 \\
Touch and Vision & 6.88 & 6.31 & 13.19 & 1.70 \\
\hline
\end{tabular}

Note-Each subject was exposed to 16 trials.

had no impact on mean accuracy scores. Although the magnitude of the effect of modality was small, a mixed-factor analysis of variance showed it to be significant $[F(2,45)=4.87, p<.05]$. However, the effect of ITI was nonsignificant $(F<1)$, as was the interaction effect $[F(2,45)=1.17, p>.05]$. With the data collapsed over ITI, a Newman-Keuls test showed that the mean number of correct bimodal judgments was greater than the mean for vision $(p<.05)$, but that the other comparisons were nonsignificant (for $\mathrm{H}+\mathrm{V}$ vs. $\mathrm{H}, \mathrm{F}=4.0, \mathrm{p}<.10$ ). A separate NewmanKeuls test performed on the means for the three modality conditions at 0 -sec ITIs showed that the bimodal mean was greater than the mean for $\mathrm{V}$ or $\mathrm{H}$ considered separately ( $p<.05$ for each comparison), but $\mathrm{V}$ and $\mathrm{H}$ were not significantly different $(\mathrm{p}>.05)$. It should be noted that almost all of the incorrect judgments consisted of the choice of the median grit value of a series.

The superiority of bimodal to visual input requires some explanation, since there are many ways in which combined input from the two senses might aid perception. First, subjects may somehow "add together" the information from the separate senses, and thereby acquire more information about texture. A similar interpretation is provided by Welch and Warren (1980) in their suggestion that a nondominant modality may aid perception by reducing uncertainty and contributing to perceptual reliability. Second, it is also possible that vision and touch normally work in a cooperative manner and that when we restrict individuals to one sense modality we deprive them of needed information.

An anomaly was found for the 280-grit paper. Subjects did not consistently judge that abrasive surface to be smoother than 240-grit paper, either for vision or touch. It is not clear what may have produced this outcome, but there are reports of similar anomalies (Bjorkman, 1967; Lederman \& Abbott, 1981).

Vision and touch produced very similar accuracy in the perception of smoothness. This finding is consistent with the results of a recent study of the perception of roughness by Lederman and Abbott (1981, Note 1), which support their contention that texture perception is accurately performed by both vision and touch. However, the present outcome is different 
from that reported by Lederman and Abbott, since they failed to find a superiority for bimodal presentations. Their failure could be the result of the procedure of obstructing vision of hand movements while subjects felt and viewed different pieces of sandpaper of the same grit value. Normal haptic interactions with objects in the environment are often coincident with visual input of the exploratory hand.

\section{EXPERIMENT 2}

The second experiment was designed to isolate the source of the benefits of multimodal perception and test the generality of the results of the first experiment. It was considered possible that the obtained superiority of bimodal input was not due to the utilization of visual and haptic texture information. An alternative possibility was that subjects based their judgments solely on vision. The opportunity to hold and manipulate the stimuli might have allowed improved illumination of the surfaces. All of the subjects in Experiment 1 were encouraged to look closely at the surfaces. The bimodal subjects, however, picked up the panels, thus bringing them closer to the overhead lighting. Therefore, illumination conditions were not equivalent for bimodal and visual input. Experiment 2 examines the factor of the opportunity to manipulate the stimuli. An additional purpose of the study was to evaluate the effect of joint visual, haptic, and auditory information on texture perception. Lederman (1979) found that while texture judgments could be based on auditory cues, subjects acted as if they did not utilize this source of information when touch was employed. It is possible, however, that subjects in the first experiment used auditory texture cues, especially since they were able to bring the stimuli rather close to their faces. Therefore, the obtained superiority of bimodal input could have been a consequence of the provision of more effective auditory cues in the bimodal condition.

Subjects in Experiment 2 examined textures with all relevant senses $(\mathrm{V}+\mathrm{H}+\mathrm{A})$, with vision and haptics $(\mathrm{V}+\mathrm{H})$, with haptics alone $(\mathrm{H})$, or with vision alone (V). The effect of holding the stimuli was also examined, since holding the stimuli might assist vision and audition.

\section{Method}

Subjects. There were 64 subjects in this experiment, none of whom was familiar with the task.

Stimull and Apparatus. Except for the omission of the panels with grit values of 280,240 , and 220 , the stimuli were identical to those in the first experiment. A duplicate set of panels identical to the first set of 14, but arranged in a different random sequence, was used. Auditory cues were attenuated with the use of acoustically isolating headphones.

Design and Procedure. The two factors were perceptual modality $(\mathrm{V}+\mathrm{H}+\mathrm{A}$ vs. $\mathrm{V}+\mathrm{H}$ vs. $\mathrm{V}$ vs. $\mathrm{H})$ and movement of the stimuli (hold vs. no hold), with repeated measures on the second factor.
All subjects were exposed to 28 trials in two blocks of 14 trials. On each trial, the subject was asked to choose the smoothest of three abrasive papers, as in the first experiment. The procedure was similar to that of Experiment 1, except that rest periods were not provided. For half of the subjects in each group, the stimuli were stationary for the first 14 trials. The subjects were then told to handle the stimuli for the last block of trials. The rest of the subjects in each group used the reverse sequence. Independent groups of subjects wore gloves and were provided with appropriate instructions for their exploration modality. All subjects were asked to point to indicate their choices.

\section{Results and Discussion}

A mixed-factor analysis of variance showed that the effect of perceptual mode was highly significant $[F(3,60)=14.15, \mathrm{p}<.01]$. Panel movement had no effect on texture perception $[F(1,60)=1.13, p>.05]$, nor was the interaction significant $(F<1)$. For the purpose of further reduction, the data were collapsed over the factor of movement and the means for the perceptual modality conditions were subjected to Newman-Keuls tests. The accuracy of relative smoothness judgments was equívalent for $\mathrm{V}+\mathrm{H}+\mathrm{A}$ and $\mathrm{V}+\mathrm{H}$, while both were superior to $\mathrm{V}$ or $\mathrm{H}$ considered separately $(p<.01)$. Further comparisons showed that $\mathrm{H}$ and $\mathrm{V}$ were not significantly different in mean performance $(p>.05)$. A control experiment showed that texture perception was equally accurate for the preferred and nonpreferred hands. ${ }^{1}$

The results were consistent with the data from Experiment 1 and demonstrated the superiority of multimodal and bimodal input (see Table 2). The superiority of combined visual and haptic information was not dependent upon panel movement. Furthermore, the auditory sense added nothing to information available from touch and vision. ${ }^{2}$ The advantage of bimodal input in Experiments 1 and 2 cannot be ascribed to the benefits provided by audition aided by movement, since the $\mathrm{V}+\mathrm{H}+\mathrm{A}$ group was comparable in performance to the $\mathrm{V}+\mathrm{H}$ group, with or without movement. The superiority of bimodal input cannot be explained in terms of illumination aided by movement, since movement provided no such benefit in this experiment. Vision and touch once again provided equal levels of performance in judgments of relative smoothness.

Table 2

Mean Number Correct for Each Modality With Movement or No Movement of the Stimuli

\begin{tabular}{llccc} 
& \multicolumn{3}{c}{ Mean Number Correct } \\
\cline { 2 - 5 } $\begin{array}{c}\text { Perceptual } \\
\text { Modality }\end{array}$ & $\begin{array}{c}\text { Move- No Move- } \\
\text { ment }\end{array}$ & ment & Total & PC \\
\hline Vision, Touch, and Audition & 12.75 & 13.13 & 25.88 & 92.4 \\
Vision and Touch & 12.75 & 12.81 & 25.56 & 91.3 \\
Touch & 11.38 & 11.56 & 22.94 & 81.9 \\
Vision & 10.75 & 11.38 & 22.13 & 79.0 \\
\hline
\end{tabular}

Note $-P C=$ total mean percent correct. 
The verbal reports and spontaneous behaviors of subjects are inconsistent with the ideas that vision is generally dominant over touch or that subjects typically add the two sources of information in making normal, multimodal texture judgments. Many subjects remarked that they tended to rely upon touch when making their judgments, since sight is often deceptive. These subjects indicated that objects in the world may appear different in texture when experienced tactually or visually. Something that looks rough could feel very smooth. Furthermore, many subjects in the bimodal conditions revealed their reliance upon touch in overt perceptual behaviors. Subjects either attempted to close their eyes or frequently tried to avert their vision from the stimuli. Few subjects actually closed their eyes, but many had to be urged to look at the stimuli while touching the abrasive surfaces. These informal observations are consistent with a couple of reported failures to obtain visual capture in texture perception studies (Fishkin, Pishkin, \& Stahl, 1975; Lederman \& Abbott, 1981). A number of researchers have argued that vision may be dominant when perception of form is required (Kaufman, 1974; Rock \& Victor, 1964; Welch \& Warren, 1980), but that such dominance need not obtain for texture, since vision and touch may be equally appropriate for texture perception (Lederman \& Abbott, 1981). It should be noted that there have been few investigations of normal cooperative behavior between the senses. The next experiment represents a formal attempt to ascertain whether visual texture is ignored when both visual and tactual texture cues are available.

\section{EXPERIMENT 3}

The aim of Experiment 3 was to explicate the nature of the superiority that has been found for joint haptic and visual input. Experiment 2 showed that the benefits of multimodal input cannot be explained through the improvement of illumination provided by the opportunity to handle the stimuli. One possible explanation is that subjects use both visual and haptic texture information in making bimodal smoothness judgments. This supposes that each sense in isolation does not provide sufficient information for accurate judgments, for, as Diderot $(1749 / 1972$, p. 80) noted: "I conclude ... that we gain much from the concerted action of the senses. To add touch to sight when vision alone is adequate, is like adding a single horse to a team of horses pulling in different directions." This explanation of the effects of multimodal input assumes that both senses provide equivalent information that can be added together to aid perceptual accuracy or reliability. However, observation of the perceptual strategies of subjects in the bimodal conditions of the first and second experiments suggested that subjects do not attend to both visual and haptic texture information when both sets of cues are available. Many subjects in the bimodal conditions seemed to rely upon touch and averted their gaze from the abrasive surfaces. Thus, it was hypothesized that subjects might ignore visual texture when haptic texture was at hand and use vision for proprioception, that is, movement control. It seemed probable that sight of hand movements was important for accurate multimodal perception.

The third experiment assesses the role of vision in bimodal perception. Subjects were asked to make relative smoothness judgments while being permitted to observe hand movements but not texture. A viewing device was used that eliminated visual texture. The subjects in the plastic view and haptic texture $(\mathrm{PV}+\mathrm{H})$ condition touched sandpaper panels while viewing the exploratory hand through plastic "stained glass." These subjects had to base their judgments of relative smoothness upon cutaneous information, with visual guidance of the hand. $\mathrm{PV}+\mathrm{H}$ subjects could not see the texture of the surface that was touched. This condition was compared with normal bimodal $(\mathrm{H}+\mathrm{V})$ exploration. The $\mathrm{H}+\mathrm{V}$ subjects can base their relative smoothness judgments upon visual texture cues and tactual texture cues with visual guidance. Certainly, if visual texture information is a contributor to the obtained superiority of bimodal input, its elimination should lead to a degradation in perceptual accuracy. Alternatively, if observers normally ignore visual texture cues and use vision for guidance of the hand, the elimination of visual texture should have no negative impact. Logically, it was necessary to ensure that the viewing apparatus made it impossible to see the textures of the abrasive papers in the experiment. Therefore, a third group of subjects was required to make relative smoothness judgments while limited to vision through the viewing device. These subjects, in the plastic view (PV) condition, were expected to operate at a chance level.

The subjects in Experiment 3 made relative smoothness judgments with normal $\mathrm{V}+\mathrm{H}$, or with vision of the exploratory hand touching surfaces, but without visual texture cues (PV $+\mathrm{H})$, or in a control condition (PV) without visual texture cues. If visual texture cues are used in bimodal judgments, $\mathrm{V}+\mathrm{H}$ performance should be better than $\mathrm{PV}+\mathrm{H}$ performance.

\section{Method}

Subjects. Ten subjects were assigned to each of the three perceptual modality conditions, for a total of 30 in all. None of the subjects had been involved in any of the previous experiments.

Stimuli and Apparatus. The stimuli were identical to the series used in the last experiment. Auditory cues were attenuated with headphones, while tactual exploration was limited to the index finger of the preferred hand with gloves. Visual texture cues were eliminated with the aid of a viewing box. The top of this box was covered by a layer of .15-cm-thick amber plastic "stained glass" (Jolite), sandwiched between two sheets of .2-cm-thick clear acrylic. The bottom of the box was covered with thin, black polyfoam. The plastic stained glass was fixed approximately 
$12.3 \mathrm{~cm}$ above the abrasive surfaces. The overall dimensions of the viewing box were $27 \times 16.5 \times 13.5 \mathrm{~cm}$. The texture of a surface can be readily seen if the plastic stained glass is placed directly upon it. However, the greater the distance between the plastic sandwich and a surface, the more difficult it is to make visual estimates of surface irregularities. Informal observation suggested that the device would effectively remove visual texture cues and make all samples of sandpaper within it indistinguishable in texture, yet permit vision of hand movements. The front of the viewing box was covered by an opaque black cloth; the back was open. It was possible to place the box in front of the large baffle used in the earlier experiments. The stimuli could then be introduced into the plastic-covered box through the aperture in the large baffle, thereby preventing subjects from ever seeing the papers.

Design and Procedure. The experiment was a simple singlefactor, between-subjects design, with the variable consisting of the mode of presentation of stimulation. The normal bimodal condition $(\mathrm{V}+\mathrm{H})$ was basically a replication of the $\mathrm{V}+\mathrm{H}$ condition of the second experiment. All subjects were exposed to 28 trials in which they had to make relative smoothness judgments. The PV subjects were asked to look at the abrasive papers through the plastic top of the viewing box and indicate a choice with a verbal response, that is, "right," "center," or "left." The PV subjects constituted a control condition and were not permitted to touch the stimuli. The subjects in the PV $+\mathrm{H}$ condition were asked to use the tip of the index finger of the preferred hand to explore the stimuli in the box, while looking at them through the stained glass sandwich top of the viewing box. This condition required haptic texture judgments of surfaces, but restricted visual input to sight of hand movements and the presence and outline of the panels. These subjects also provided a verbal response but, like the others, were not given feedback. The subjects were not permitted to manipulate the panels within the plastic-covered box. The basic difference between the $\mathrm{PV}+\mathrm{H}$ condition and the normal $\mathrm{V}+\mathrm{H}$ condition consists of the elimination of visual texture cues by the plastic stained-glass top of the viewing box. As in Experiment 2, the order of presentation of the 28 panels was randomized and rest periods were not provided. The task was like that of the second experiment, with the major difference being the modification of visual input in the $P V$ and $P V+H$ conditions.

\section{Results and Discussion}

The mean number correct for the bimodal $\mathrm{V}+\mathrm{H}$, $\mathrm{PV}+\mathrm{H}$, and $\mathrm{PV}$ conditions were $26.3,25.7$, and 7.8 , respectively. The standard deviations for the $\mathrm{V}+\mathrm{H}$, $\mathrm{PV}+\mathrm{H}$, and PV conditions were $1.74,1.49$, and 1.89 , respectively. The elimination of visual texture information had no deleterious effect on bimodal judgments. It should be noted that these bimodal judgments are comparable to the scores for the $\mathrm{V}+\mathrm{H}$ condition in the second experiment. Furthermore, the plastic cover on the viewing device effectively eliminated visual texture, since PV performance was slightly lower than chance at $27.9 \%$ (chance probability $=33.3 \%$ ). This means that subjects in the bimodal condition do not simply add visual and tactual texture information when making judgments of relative smoothness. If that were the case, subjects denied visual texture with the viewing box should have performed like the haptic subjects in the second experiment. The haptic subjects in Experiment 2 performed at a lower level than the bimodal subjects. Thus, a ceiling effect could not be operating in Ex- periment 3 , since lower performance levels have been obtained with the same set of stimuli when observers were restricted to either sense in isolation. A one-way analysis of variance showed that perceptual mode had a significant effect on mean performance $[F(2,27)$ $=340.0, \mathrm{p}<.011$. A Newman-Keuls test indicated that the two bimodal conditions were not significantly different $(p>.05)$, but both were superior to the PV condition $(\mathrm{p}<.01)$.

Normal observers act as if they ignore visual texture information when both haptic and visual cues are available. If bimodal superiority cannot be atributed to the aid provided by visual texture cues, its effectiveness is probably due to the opportunity to observe hand movements. Vision aids in information pickup by acting as touch's servant. It provides proprioceptive feedback on exploratory activity to permit the skin to maximize information pickup. Accurate texture perception may require controlled hand movements, which could be lacking when observers are asked to "grope in the dark." To perceive accurately the difference in smoothness between two surfaces, one must apply the same movement sequence and amount of pressure to each surface. It is suggested that vision and touch normally function in a cooperative manner and are mutually interdependent. Perception is facilitated when the senses aid each other.

\section{GENERAL DISCUSSION}

The results of the experiments suggest that texture perception may normally be multimodal. The restriction of an observer to a single modality degrades performance in texture-perception tasks. This is indicated by the superiority of bimodal input to either visual or haptic input presented individually. Furthermore, the superiority of bimodal experience may be the result of a "division of labor" between the senses. While subjects use both vision and touch in making bimodal texture judgments, their contributions are not identical. The tactual sense is relied upon for information about surface texture, while vision provides control over hand movements. Such a conclusion is supported by the results of Experiment 3, since the elimination of visual texture does not impair performance for bimodal conditions when visual regulation ${ }^{3}$ is still provided. It is proposed that visual guidance assists the active observer in haptic explorations of the environment. The results of the third experiment are difficult to explain unless the assumption is made of some differentiation in functioning for vision and touch.

Haptic cues may be more perceptually salient than visual texture cues when both sources of information are available and redundant. The results of Experiment 3 showed that subjects seem to ignore visual 
texture in making bimodal judgments. People may have learned that visual texture does not provide reliable information about surface irregularities and consequently depend upon touch. We have often experienced surfaces that are featureless to touch, but provide visual texture information. The clearest example of this might be a photograph or a painting. The attentional habits of the subjects in this study also suggest a reliance upon touch. The subjects in the bimodal conditions of ten had to be urged to look at the stimuli. It is suggested that these subjects may have been attempting to use peripheral vision to monitor their hands and not the surfaces being touched. Perhaps observation of the hand precludes attention to both haptic and visual texture.

The experiments generally show that both vision and touch perform at similar levels of accuracy when relative smoothness judgments are required. The senses certainly work most efficiently together and the visually guided hand is superior to either sense considered separately. Furthermore, subjects seem to show a preference for tactual texture cues when vision is available for guidance of the hand. It must be admitted that there is more to the world of texture than abrasive papers. There are obviously both finer and coarser textures than those employed in the present study. It is not known if vision and touch will prove equally accurate given a different range of stimuli.

\section{REFERENCE NOTE}

1. Lederman, S. J., \& Abbott, S. G. Texture perception: Studies of intersensory organization using a discrepancy paradigm, and visual vs. tactual psychophysics. Paper presented at the meetings of the Psychonomic Society, St. Louis, Missouri, November 1980.

\section{REFERENCES}

Bjorkman, M. Relations between intra-modal and cross-modal matching. Scandinavian Journal of Psychology, 1967, 8, 65-76.

Dide Rot, D. Letter on the blind. In M. Jourdain (Ed. \& trans.), Diderot's early philosophical works. New York: Burt Franklin, 1972. (Originally published, 1749.)

Fishkin, S. M., Pishkin, V., \& Stahl, M. L. Factors involved in visual capture. Perceptual and Motor Skills, 1975, 40, 427-434.

Fraser, A. B. The psychological foundation of natural realism. American Journal of Psychology, 1891, 4, 429-450.

Freides, D. Human information processing and sensory modality: Cross-modal functions, information complexity, memory, and deficit. Psychological Bulletin, 1974, 81, 284-310.

Gibson, J. J. Observations on active touch. Psychological Review, $1962,69,477-491$.

Gibson, J. J. The useful dimensions of sensitivity. American Psychologist, 1963, 18, 1-15.

GiBson, J. J. The senses considered as perceptual systems. Boston: Houghton Mifflin, 1966.

Gisson, J. J. The ecological approach to visual perception. Boston: Houghton Mifflin, 1979.

Kaufman, L. Sight and mind. New York: Oxford University Press, 1974.

Lederman, S. J. Auditory texture perception. Perception, 1979, 8, 93-103.
Lederman, S. J., \& Aвbott, S. G. Texture perception: Studies of intersensory organization using a discrepancy paradigm and visual vs. tactual psychophysics. Journal of Experimental Psychology: Human Perception and Performance, 1981, 7, 902-915.

LEE, D. N. The functions of vision. In H. L. Pick \& E. Saltzman (Eds.), Modes of perceiving and processing information. Hillsdale, N.J: Erlbaum, 1978.

Mommers, M. J.C. Braille reading: Effects of different hand and finger usage. Journal of Visual Impairment and Blindness, $1980,74,338-343$.

NeISSER, U. Cognition and reality. San Francisco: Freeman, 1976.

REID, T. Inquiry into the human mind. In Sir William Hamilton (Annotator), Thomas Reid: Philosophical works (Vol. 1). Hildesheim, Germany: George Olms, 1967. (Originally published, 1764.)

Rock, I., \& Victor, J. Vision and touch: An experimentally created conflict between the two senses. Science, 1964, 143, 594-596.

Rudel, R. G., \& Teuber, H.-L. Crossmodal transfer of shape discrimination by children. Neuropsychologia, 1964, $2,1-8$.

Smith, J. M. The sensory functions of the non-preferred hand. Journal of Experimental Psychology, 1934, 17, 154-159.

Stevens, S. S., \& Harris, J. R. The scaling of subjective roughness and smoothness. Journal of Experimental Psychology, $1962,64,489-494$.

Taylor, M. M., Lederman, S. J., \& Gibson, R. H. Tactual perception of texture. In E. C. Carterette \& M. P. Friedman (Eds.), Handbook of perception (Vol. 3): Biology of perceptual systems. New York: Academic Press, 1974.

von Fieandi, K. Toward a unitary theory of perception. Psychological Review, 1958, 65, 315-320.

Walker, J. T. Successive contrast: A tactual after-effect. American Journal of Psychology, 1966, 79, 328-329.

WELCh, R. B., \& WARREN, D. H. Immediate perceptual response to intersensory discrepancy. Psychological Bulletin, 1980, 88, 638-667.

\section{NOTES}

1. A control experiment was performed to evaluate the effect of the use of the preferred hand on texture perception. It is possible that the preferred hand is less efficient than the nonpreferred hand for information pickup (Mommers, 1980; Smith, 1934). Twelve right-handed subjects were exposed to the stimuli of Experiment 2 and required to make relative smoothness judgments with either the left or right hand. The hand factor was completely counterbalanced, with repeated measures on each subject. It did not matter which hand touched the abrasive surfaces $(t<1)$. In terms of the number of correct responses, the means for the left and right hands were 11.92 and 11.83 , respectively. Performance for touch was comparable to that in Experiment 2. Therefore, the results of the experiments could not have been biased through a poor choice of the preferred hand for haptic conditions.

2. An additional control experiment was performed to determine if auditory cues could have been utilized by subjects in the first experiment, in which audition was uncontrolled. Subjects were asked to make relative smoothness judgments with touch alone or with both touch and audition. The 12 subjects were exposed to 28 trials and the same stationary stimuli used in Experiments 2 and 3 and described in Footnote 1. The presence of audition provided no assistance to touch, since the means for touch alone and bimodal presentations were 11.58 and 11.5 , respectively.

3. Gibson $(1963,1966,1979)$ and Lee $(1978)$ have described the importance of vision for the control of purposive behaviors. Accurate texture perception can be viewed as the achievement of an active perceiver. Vision may also subserve manual exploration of other surface characteristics.

(Manuscript received March 12, 1981; revision accepted for publication December 7, 1981.) 